\title{
Analisis de las principales variables de proceso que influyen en el rechazo de los cátodos durante el electrorrefino del cobre $e^{(\cdot)}$
}

\author{
G. Cifuentes*, C. Vargas* y J. Simpson* \\ Resumen \\ Se trabajó en un circuito experimental de electrorrefinación de cobre, utilizando tres tipos de ánodos procedentes \\ de tres fundiciones chilenas diferentes: Hernán Videla Lira, Las Ventanas y El Teniente. En este circuito, se estudió \\ el problema del rechazo de cátodos y la influencia de algunas variables de proceso en este fenómeno. Las variables ana- \\ lizadas fueron: flujo de electrolito en la celda, sólidos en suspensión, densidad de corriente, dopado de plomo en los \\ ánodos, densidad de los barros anódicos generados y tamaño de partícula de los barros anódicos. Los principales re- \\ sultados obtenidos a partir del estudio del circuito experimental fueron los siguientes: el flujo de electrolito no afec- \\ ta significativamente el rechazo de cátodos, a medida que aumenta la densidad de corriente disminuyen los rechazos, \\ el aumento de los sólidos en suspensión provoca cátodos fuera de norma y a mayor cantidad de plomo en los ánodos \\ menores fueron los rechazos.
}

\section{Analysis of the process main variables influence in the rejection of the cathodes during copper electrorefining}

\begin{abstract}
An experimental circuit of copper electrorefining using three types of anodes coming from three different Chilean foundries: Hernán Videla Lira, Las Ventanas and El Teniente was used to simulate the electrorefining process. In this circuit the problem of the cathode rejection and the influence of some process variables in this phenomenon were studied. The variables analyzed were: electrolyte cell flow, solids in suspension, current density, lead doping in the anodes and density and particle size of the anodic slimes generated. The main results obtained from the experimental circuit were the following: the electrolyte flow doesn't affect significantly the cathodic rejection, an increase of current density produces a decrease of cathodic rejections, the presence of the solids in suspension causes cathodes outside of norm, and to bigger quantity of lead in the anodes smallest were the rejections.
\end{abstract}

Keywords Copper; Electrorefining; Cathodic rejection.

\section{INTRODUCCION}

La refinación electrolítica es el último proceso metalúrgico al que es sometido el cobre procedente de materias primas sulfuradas y, por tanto, reviste características especiales de calidad. La electrorefinación es utilizada para producir cátodos de cobre de alta calidad a partir de ánodos que contienen una variedad de impurezas. Este proceso es llevado a cabo en un electrolito acuoso que contiene acido sulfúrico y sulfato de cobre principalmente, a una temperatura cercana a os $65^{\circ} \mathrm{C}$. Durante la electrólisis, los ánodos se disuelven y se deposita cobre metálico sobre el cá- todo. Los cátodos de cobre deben reunir criterios de calidad tales como un depósito catódico liso (sin presencia de nódulos ni dendritas), un bajo contenido de impurezas (99,99 \% de pureza) y un bajo nivel de porosidad. Los depósitos lisos, sin nódulos y no porosos contienen muy pocas impurezas. Estos, además, deben ser obtenidos bajo altas eficiencias de corriente $(>90 \%)$. Esta es una tarea compleja ya que el crecimiento de los cátodos está influenciada por muchos parámetros tales como densidad de corriente, temperatura del electrolito, composición del electrolito, composición del ánodo, espaciamiento ánodo-cátodo, flujo de electrolito y probablemente una de las

\footnotetext{
(•) Trabajo recibido el día 23 de mayo de 2007 y aceptado en su forma final el día 7 de enero de 2009.

* Departamento de Ingeniería Metalúrgica, Facultad de Ingeniería, Universidad de Santiago de Chile (USACH), Avenida Libertador Bernardo O’Higgins 3363, Casilla 10233, Fono- Fax: 56-2-7183224, Santiago, Chile. E-mail: gerardo.cifuentes@usach.cl.
} 
más importantes: la dosificación y concentración de $\operatorname{aditivos}^{[1]}$.

La calidad del cobre electrolítico es una de las mayores preocupaciones de las empresas refinadoras de cobre y, de esta manera, los niveles de impurezas adquieren una gran importancia. Algunos de los efectos dañinos que tienen las impurezas sobre las propiedades físicas del cobre son disminución de su conductividad, ablandamiento, destemple y efecto en la temperatura de recristalización ${ }^{[2 \text { y 3]. }}$.

La producción de cátodos de cobre de alta pureza es el objetivo principal de las refinerías de cobre a pesar del aumento, en cantidad y tipo, de las impurezas de los ánodos que son electrorrefinados. La problemática de las impurezas presentes en el proceso de electrorefinación de cobre, se traduce en costos directos para la empresa debido a que se producen cátodos físicamente irregulares, los cuales son rechazados por no ser aptos para su comercialización. Los cátodos de cobre que no cumplen con las normas de calidad exigidas para su comercialización son rechazados y recirculados a las operaciones previas de fundición. Esto implica una disminución en la capacidad productiva y un aumento en la carga circulante de impurezas en el sistema. Esta acción se denomina "rechazo catódico" y se evalúa como un porcentaje de la producción total de cátodos de cobre.

Se han publicado diversos estudios con respecto al comportamiento e influencia de impurezas en la calidad catódica ${ }^{[4-7]}$, pudiéndose resumir tales trabajos en lo siguiente:

- Los elementos menos nobles que el cobre, como cinc, níquel y hierro se disuelven fácilmente en el electrolito y permanecerán en él hasta que sean removidos por el electrolito de descarte.

- Los elementos más electropositivos que el cobre, como el selenio, telurio, plata, oro, paladio, platino y elementos que son insolubles en ácido sulfúrico, como el plomo, formarán compuestos sólidos insolubles que caerán del ánodo al fondo de las celdas formando el denominado "barro anódico" [8 y 9].

- Un tercer grupo de elementos comprende las impurezas que tienen un potencial de disolución comparable al cobre, como el arsénico, antimonio y bismuto. Estos elementos se comportan de diferentes maneras, dependiendo de la composición del ánodo y de otros parámetros operacionales. De acuerdo a esto, es posible encontrar estos elementos en el electrolito, formando compuestos sólidos finos y/o densos, con un modelo fluctuante de distribución. Estos elementos, dependiendo de su concentración en disolución, pueden formar un amplio rango de compuestos insolubles, denominados "lamas flotantes", que están formadas de arseniato de antimonio, $\mathrm{SbAsO}_{4}$, y arseniato de bismuto, $\mathrm{BiAsO}_{4}$. Se forman por la precipitación a partir del ánodo o del electrolito y pueden flotar en la superficie de éste pudiendo contaminar el cátodo.

Las impurezas pueden provocar los siguientes problemas en el proceso de electrorefinación:

- Producción de barros anódicos, los cuales se desplazan hacia el cátodo por convección, siendo ocluidos aquí mecánicamente.

- Se pueden formar precipitados, como $\mathrm{SbAsO}_{4}$, en el seno del electrolito que acaban incorporándose por oclusión mecánica al cátodo.

- El electrolito contiene impurezas disueltas las cuales pueden ser atrapadas en microespacios producidos entre los cristales de cobre en la superficie del cátodo.

- La presencia de algunas impurezas en disolución, las cuales pueden ser depositadas junto al cobre, contaminando el producto final.

Aunque el proceso de electrorefinación de cobre está bien establecido y es altamente eficiente, no está libre de ciertos problemas operacionales. Uno de los problemas persistentes en operaciones de electrorrefino del cobre es el crecimiento de nódulos en los cátodos de cobre, causado principalmente, al parecer, por partículas suspendidas en el electrolito. La inclusión de partículas sólidas en la deposición catódica es una de las principales causas de crecimiento de dendritas en el cátodo durante electrorefinación de cobre ${ }^{[10-}$ 12]. Cuando partículas extrañas están en contacto con la superficie de crecimiento de cristales de cobre, sirven como substratos para la nucleación bidimensional (2D) ${ }^{[12]}$. Consecuentemente, la velocidad de crecimiento local de la superficie será incrementada y comienza a generarse un grano rugoso. La generación de una nueva capa tiene lugar a través de un proceso de nucleación 2D el cual se manifiesta por una formación de cristales alargados en la dirección del campo eléctrico. Dependiendo de su cantidad y tamaño, la presencia de partículas sólidas en los bordes de grano puede causar inestabilidad superficial de la capa creciente y provocar un crecimiento desorientado de los cristales ${ }^{[12]}$. Estos mecanismos dan origen al crecimiento de dendritas. En bajas cantidades, la presencia de sólidos puede solamente resultar en un grano rugoso sin un crecimiento significativo de la dendrita.

Las partículas solidas presentes en el electrolito de cobre pueden tener su origen desde tres fuentes 
principales: desde el ánodo, desde el barro anódico y desde el material que se utiliza para recubrir la superficie del molde de ánodos. Las partículas sólidas que se originan desde el ánodo son, por ejemplo, plomo como $\mathrm{PbSO} 4, \mathrm{PbO}$, así como óxidos mixtos de $\mathrm{Pb}-\mathrm{As}-\mathrm{Cu}$. La cantidad de estos sólidos en el electrolito es dependiente del contenido de los elementos respectivos en el ánodo. Parte de estas partículas solidas provenientes del ánodo son transportadas al barro anódico y otras pueden ir al electrolito.

Además de la parte principal que está cayendo al fondo de la celda, algo de las partículas de barro anódico están suspendidas en el electrolito. Consecuentemente, las condiciones a las cuales se produce la suspensión de las partículas, tales como aumento de densidad o viscosidad del electrolito, la evolución de gases en el ánodo y una alta caída de barros incrementará, indirectamente, la susceptibilidad del crecimiento de dendritas en el cátodo ${ }^{[13]}$. Bajo ciertas condiciones, también se pueden formar lamas flotantes cerca del nivel superficial del electrolito ${ }^{[14]}$.

En la operación industrial de electrorefinación de cobre se añaden aditivos al electrolito, tales como tiourea $(\mathrm{TU})$, gelatina $(\mathrm{G})$ y cloruro $(\mathrm{Cl})$ para mantener la calidad catódica y promover un depósito catódico liso ${ }^{[15]}$. Estos aditivos, que actúan mayoritariamente como inhibidores, se adsorben sobre la superficie del cátodo donde toman parte en el proceso de electrocristalización ${ }^{[16]}$. Se encuentra claramente establecida la necesidad de aditivos para la deposición catódica, aunque su mecanismo de acción durante la electrolisis no está completamente comprendido ${ }^{[16]}$. Sin embargo, los aditivos pueden también facilitar la formación de nódulos cuando sus concentraciones son inadecuadas ${ }^{[17}$ y 18$]$.

$\mathrm{Si}$ bien es cierto que son numerosos los estudios acerca de las variables que influyen en la calidad del cátodo, hoy en día, se requiere realizar la electrorefinación de cobre a altas densidades de corriente para mejorar la productividad de las refinerías. Sin embargo, surgen diversos problemas cuando se incrementa la densidad de corriente. La principal problemática consiste en una disminución en la calidad de cátodo, con el consecuente rechazo, y en la eficiencia de corriente, debido, probablemente, al fenómeno de nodulación. Manteniendo todas las demás variables constantes, con dosificaciones y concentraciones de aditivos que producen un cátodo de buena calidad bajo una menor densidad de corriente, no son necesariamente adecuadas bajo una mayor densidad de corriente. De hecho, ellas, pueden aun disminuir la calidad del cátodo y causar nodulación. Incrementar la densidad de corriente sin disminuir la calidad catódica es uno de los problemas para las refinerías de cobre.
Esta situación ha dado paso, en los últimos años, a una mayor necesidad en la vigilancia o control de las operaciones de electrorefinación y a estudios tendentes a comprender, en mejor forma, el comportamiento de las impurezas y a determinar cuáles deben ser las medidas a tomar para el control de aquellas en el proceso electrolítico.

De esta manera, el objetivo principal de este trabajo ha sido determinar la influencia de las principales variables de proceso sobre la calidad física de los cátodos, trabajando en un circuito industrial experimental para, así, poder establecer las causas que llevan a la aparición del rechazo de éstos.

\section{TRABAJO EXPERIMENTAL}

Se trabajó en un circuito experimental de la Refinería Electrolítica de Las Ventanas ubicada en la Región de Valparaíso, Chile. Este circuito estaba conformado por 8 celdas industriales, cada una con 32 cátodos. Se utilizaron tres tipos de ánodos: HVL, Ventanas y Teniente, cuya composición química se presenta en la tabla I. Se trabajó en condiciones lo más parecidas posible a las de los circuitos comerciales, esto es, flujo de $18 \mathrm{l} / \mathrm{min}$, temperatura de entrada de $66^{\circ} \mathrm{C}$ y $64^{\circ} \mathrm{C}$ de salida, 43 a $46 \mathrm{~g} / \mathrm{l}$ de cobre, 176 a $180 \mathrm{~g} / \mathrm{l}$ de ácido sulfúrico y ciclo anódico de 16 días. El volumen total de electrolito presente en el circuito experimental fue de, aproximadamente, $44 \mathrm{~m}^{3}$, donde $10 \mathrm{~m}^{3}$ corresponden al estanque de recirculación.

La densidad de corriente de trabajo fue variable, utilizándose 250,290 y $310 \mathrm{~A} / \mathrm{m}^{2}$ para los tres tipos de ánodos con el objetivo final de analizar las características físicas de los cátodos producidos.

El flujo de trabajo normal, de ingreso de electrolito a las celdas, en la nave electrolítica es de 18 $1 / \mathrm{min}$. En el circuito experimental, este flujo se varió hasta un valor mínimo de 12 y un valor máximo de 30 1/min; esto se realizó sólo en una de las ocho celdas.

Además, para los tres tipos de ánodos, se efectuó un dopado de plomo, previo a la etapa de moldeo, con el objeto de estudiar el efecto que pudiera tener este elemento sobre los rechazos de los cátodos y la composición del barro anódico. Este dopado consistió en adicionar una cantidad determinada de plomo en la etapa de reducción del proceso de fundición; dicha cantidad dependería de la concentración de plomo que se quisiera obtener en el ánodo moldeado.

Los métodos de análisis y/o de medición utilizados fueron los siguientes: flujo de electrolito por medio de flujómetro; sólidos en suspensión a través de filtrado y posterior pesada; densidad de corriente 
ANALISIS DE LAS PRINCIPALES VARIABLES DE PROCESO QUE INFLUYEN EN EL RECHAZO DE LOS CÁTODOS DURANTE EL ELECTRORREFINO DEL COBRE ANALYSIS OF THE PROCESS MAIN VARIABLES INFLUENCE IN THE REJECTION OF THE CATHODES DURING COPPER ELECTROREFINING

Tabla I. Composición química de los ánodos de cobre estudiados

Table I. Chemical composition of the studied copper anodes

\begin{tabular}{cccc}
\hline Elemento (\%) & Ánodo HVL (mg/l) & Ánodo Ventanas $(\mathrm{mg} / \mathrm{l})$ & Ánodo Teniente (mg/l) \\
\hline $\mathrm{Cu}$ & 99,61 & 99,60 & 99,62 \\
$\mathrm{As}$ & 292,48 & 808,44 & 917,40 \\
$\mathrm{Sb}$ & 52,61 & 366,38 & 140,95 \\
$\mathrm{~Pb}$ & 652,25 & 321,8 & 37,9 \\
$\mathrm{Te}$ & 30,33 & 53,1 & 10,3 \\
$\mathrm{Se}$ & 160,5 & 201 & 218,08 \\
$\mathrm{Ni}$ & 355,00 & 256,72 & 138,4 \\
$\mathrm{Fe}$ & 75,75 & 44,8 & 12,65 \\
$\mathrm{Bi}$ & 5,38 & 8,14 & 3,45 \\
$\mathrm{Ag}$ & 407,42 & 477,2 & 133,5 \\
$\mathrm{Au}$ & 29,61 & 22,84 & 1,20 \\
\hline
\end{tabular}

catódica por medio de amperímetro; dopado de plomo en los ánodos por análisis de espectroscopia de absorción atómica; análisis de densidad de los barros anódicos generados por picnometría; y análisis granulométrico de los barros anódicos por Laser Sizer.

\section{RESULTADOSY DISCUSION}

\subsection{Flujo de electrolito}

Ya que un aumento en el flujo de electrolito se traduce en una mayor agitación de éste, al trabajar con flujos mayores a $18 \mathrm{l} / \mathrm{min}$ el número de cátodos rechazados debería aumentar, a causa de la mayor probabilidad de formación de nódulos sobre su superficie por las partículas sólidas procedentes del barro levantado por la agitación. De igual forma, los sólidos en suspensión medidos deberían aumentar. Sin embargo, en las campañas en las que se varió el flujo no se observó un aumento en los sólidos en suspensión ni en los rechazos de cátodos de la celda en estudio, a pesar de haber mantenido un estricto control sobre la condición en estudio durante todo el ciclo anódico. Esta respuesta va en contra de lo esperado.

Por lo tanto, se puede deducir que el hecho de aumentar el flujo de electrolito, sólo en una de las celdas del circuito experimental, no provoca más rechazos (en la celda en cuestión) que al trabajar con un flujo normal de $18 \mathrm{l} / \mathrm{min}$. Lo cual se debe, tal vez, a que existe mayor incidencia sobre los rechazos cuando el flujo ingresado a las celdas contiene una mayor cantidad de partículas sólidas. Esto es así cuando el electrolito que contiene el estanque de recirculación se ve perturbado, ya sea por traspaso de éste desde otros circuitos o por variaciones en la bomba centrífuga que lleva el electrolito a las celdas. De esta forma, aún manteniendo el flujo en niveles elevados (30 1/min) el sistema permanecerá "calmado" (mientras no haya perturbaciones externas) existiendo una menor cantidad de partículas que ingresen a las celdas. A pesar de promoverse una agitación por movimiento del electrolito, parece que esto no es un hecho lo suficientemente importante como para provocar que las partículas se ocluyan en el cátodo.

Por otro lado, tampoco se observó ningún efecto apreciable al utilizar flujos menores a $18 \mathrm{l} / \mathrm{min}$, ni en los rechazos de cátodos ni en los sólidos en suspensión.

\subsection{Sólidos en suspensión}

En términos generales, se encontró que a medida que transcurre el tiempo, durante el ciclo anódico, los sólidos en suspensión disminuyen, pudiéndose suponer que el sistema trabaja como un decantador de partículas. Cualquier perturbación externa que provoque el movimiento de las partículas, se traduce en un aumento sobre los rechazos de cátodos por oclusión de sólidos sobre la superficie del cátodo. Se esperaba que a medida que pasaran los días los sólidos aumentarían, situación que se tornó contraria. Además, en las campañas en las que hubo evacuación de electrolito al término del primer ciclo catódico, se observó que, al comienzo del siguiente ciclo, los sólidos aumentaron nuevamente, tendiendo a disminuir con los días para, después, mostrar un leve aumento al término del ciclo anódico total. Para aquellas campañas en las que se mantuvo el 
electrolito en las celdas, desde el inicio hasta el final del ciclo anódico, fue más claro el hecho de observar una disminución de los sólidos a medida que pasaban los días, prácticamente desde $6 \mathrm{mg} / \mathrm{l}$ hasta 2-1.5 mg/l, al igual que se notó un leve aumento al final del proceso. La variación de los sólidos en suspensión a través del tiempo, para los tres tipos de ánodos estudiados, se muestra en la figura 1 .

Cabe señalar que, cada vez que existía traspaso de electrolito desde otros circuitos al circuito experimental, los sólidos en suspensión aumentaban, del mismo modo que lo hacía cuando ocurría un corte energético o cuando los electrodos estaban sometidos a movimientos constantes (cuando se realizaba una inspección muy "fuerte").

De acuerdo al comportamiento de los sólidos en suspensión, se puede decir que las celdas del circuito experimental actúan como un sistema de decantación de sólidos, permitiendo que los mismos se mantengan en el fondo de la celda, salvo que exista alguna perturbación externa que los levante. Se debe mencionar que estos sólidos están relacionados directamente con los barros anódicos o lamas flotantes pues son los más fáciles de ser puestos en movimiento cuando ocurre algún tipo de agitación.

El hecho que al final del ciclo anódico se observe un aumento en la medición de sólidos podría señalar que la superficie del ánodo comienza a transformarse formándose una capa, cada vez más porosa a medida que transcurre el tiempo y llegando a ser es tan gruesa y pesada que no es posible mantenerla adherida al cuerpo del ánodo cayendo; así, al fondo de la celda; esto, provoca agitación y levantamiento del barro ya decantado y, por ende, un aumento en los sólidos presentes en el electrolito.
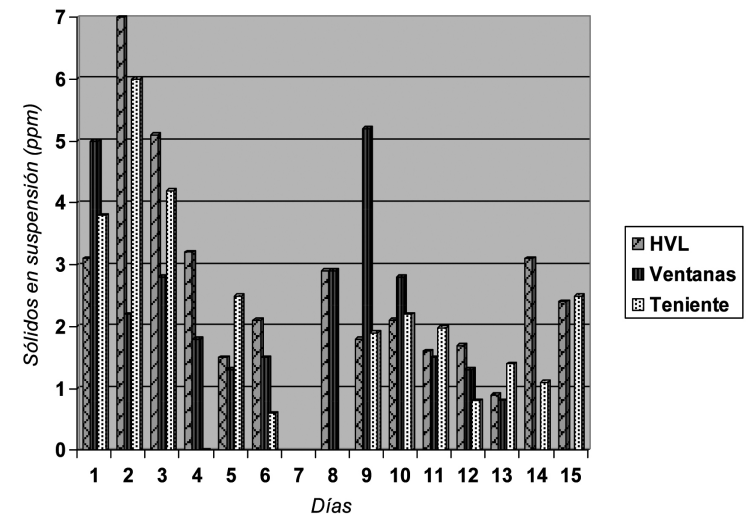

Figura 1. Sólidos en suspensión para ánodos HVL, Ventanas y Teniente.

Figure 1. Solids in suspension for HVL, Ventanas and Teniente anodes.

\subsection{Densidad de corriente}

Para la densidad de corriente de trabajo, la figura 2 permite establecer que, a medida que esta variable aumenta, los nódulos formados en la superficie del cátodo disminuyen y, por tanto, el rechazo físico; eso si, manteniendo un riguroso control sobre los aditivos. Esta disminución de los rechazos podría tener su explicación en los resultados de los análisis de tamaño de partícula de los barros anódicos generados. La figura 3 muestra que mientras más altas fueron las

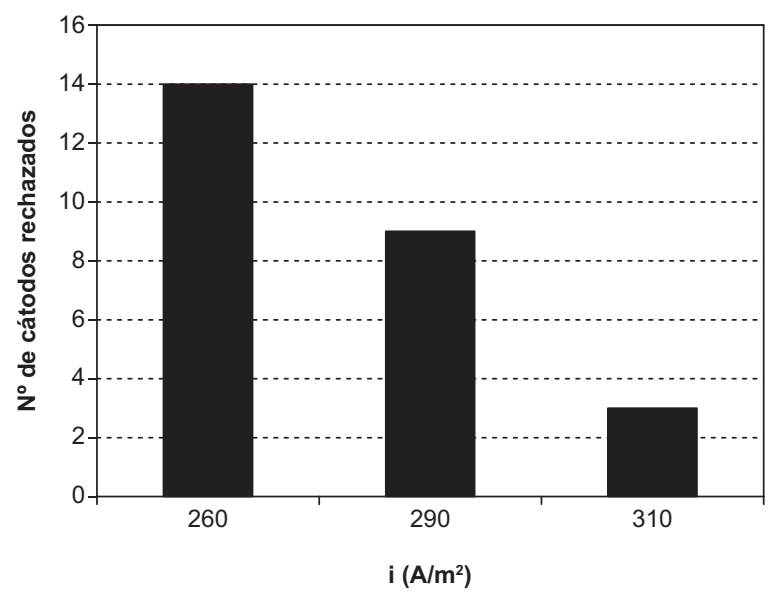

Figura 2. Influencia de la densidad de corriente sobre el rechazo de cátodos.

Figure 2. Current density influence on cathode rejection.

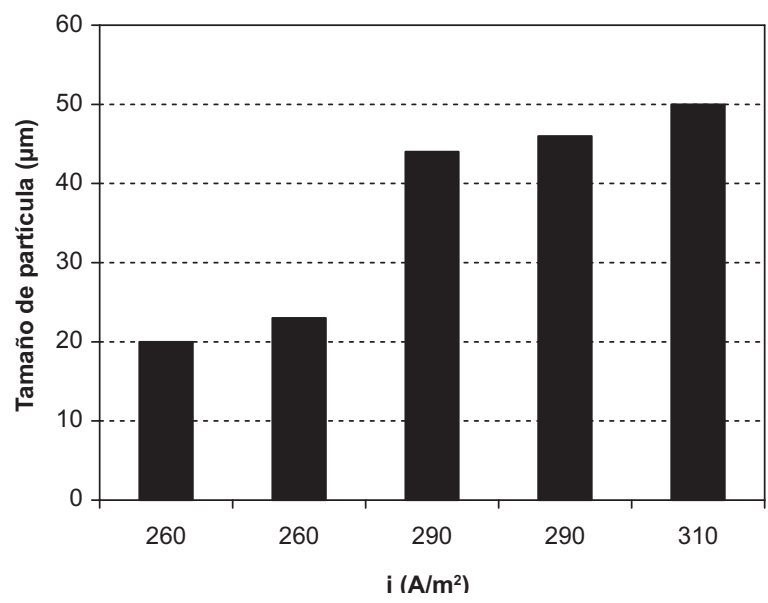

Figura 3. Influencia de la densidad de corriente sobre el tamaño de partícula de los barros anódicos.

Figure 3. Current density influence on particle size of anodic slimes. 
densidades de corriente, el tamaño de partícula de los barros anódicos resultó ser mayor, influyendo, directamente, sobre la disminución del rechazo de cátodos (físico). Esta disminución se debería a que un aumento en el tamaño de las partículas de barro anódico permite una mayor velocidad de sedimentación de éste en el sistema.

Teóricamente, al aumentar la densidad de corriente aumentará la producción catódica, debido a la ley de Faraday. Sin embargo, el trabajar con densidad de corriente alta disminuye la calidad morfológica del cátodo pues el depósito es más granular o pulverulento, con la consiguiente formación de nódulos, causantes de cortes y de los rechazos. Es aquí donde los aditivos orgánicos juegan un papel de importancia al mantener las condiciones adecuadas para un depósito óptimo, debiendo existir un control sobre la concentración y dosificación de éstos al sistema. Al utilizar una densidad de corriente determinada, la concentración de aditivos estará controlada por su magnitud. Esto es, mientras mayor sea la densidad de corriente mayor debería ser la concentración de aditivos orgánicos para compensar los efectos que produzca la velocidad de deposición de cobre sobre la superficie de los cátodos. Lo anterior se explica en el contexto de cómo actúan los aditivos en la electrorrefinación de cobre. La cola (polímero superior proteínico de cadenas pépticas CO-N, de peso molecular $15.000 \mathrm{~g} / \mathrm{mol}$ ), la tiourea y el avitone (un tipo de poliacrilamida) son compuestos polarizantes catódicos, los dos primeros, mientras el último actúa como floculante de sólidos en suspensión. Los aditivos polarizantes se agregan en dosis aproximadas de 2 a $4 \mathrm{mg} / \mathrm{l}$ en el electrolito y, básicamente debido a su alto peso molecular, son adsorbidos superficialmente permitiendo controlar las imperfecciones cristalinas en el cátodo. Si estas imperfecciones estructurales en la superficie del cátodo se controlan correctamente, será posible producir un cobre de la alta calidad a altas densidades de operación. La calidad del metal depositado en el cátodo es afectada, apreciablemente, por la estructura real del depósito. Hay indicaciones de que la cantidad de impurezas en la estructura del metal depositado depende grandemente de la textura del metal depositado. Cuanto más densa es la estructura del metal depositado y la superficie del cátodo es más lisa, más baja es la cantidad de impurezas fijadas por adsorción durante la deposición del metal.

En muchas de las campañas estudiadas, el control sobre los aditivos orgánicos fue deficiente, permitiéndose su degradación al alcanzarse temperaturas demasiado elevadas. A la vez, se tuvo una dosificación inadecuada de acuerdo con la densidad de corriente utilizada, provocando una calidad morfológica de los cátodos deficiente. Por ello, se hace hincapié en el hecho que, en general, al utilizar densidades de corriente altas, los rechazos físicos son más bajos con una morfología nodular mínima y aceptable, que permite su posterior comercialización siempre que se tenga un estricto control sobre la cantidad de aditivos orgánicos.

Por tanto, se puede concluir que al aumentar la densidad de corriente de trabajo disminuyen los nódulos dispersos en la superficie del cátodo y, así, el rechazo físico, con la condición de mantener un riguroso control sobre los aditivos. Esta disminución de los rechazos podría tener su explicación en los análisis de tamaño de partícula de los barros anódicos generados, cuya descripción se hará más adelante.

\section{4. "Dopado" de plomo en los ánodos}

El efecto del "dopado" con plomo en los ánodos se presenta en la figura 4. La presencia de plomo en los ánodos favorecería la disminución de antimonio en el electrolito, permitiendo que éste decantase junto al plomo minimizando la probabilidad de formación de nódulos en los cátodos por oclusión de partículas sólidas (antimonio, en este caso) presentes en el electrolito. Se pudo constatar la tendencia de que a medida que la cantidad de plomo presente en los ánodos era mayor, los rechazos disminuían.

Es sabido que existen sólidos en suspensión o lamas flotantes que no sedimentan, manteniéndose en el electrolito y siendo causantes de la formación de nódulos por oclusión de estos compuestos sobre la superficie de los cátodos. Estos compuestos son de arsénico y de antimonio, principalmente. Ahora bien, algunas investigaciones ${ }^{[19}$ y 20$]$ han señalado la existencia de

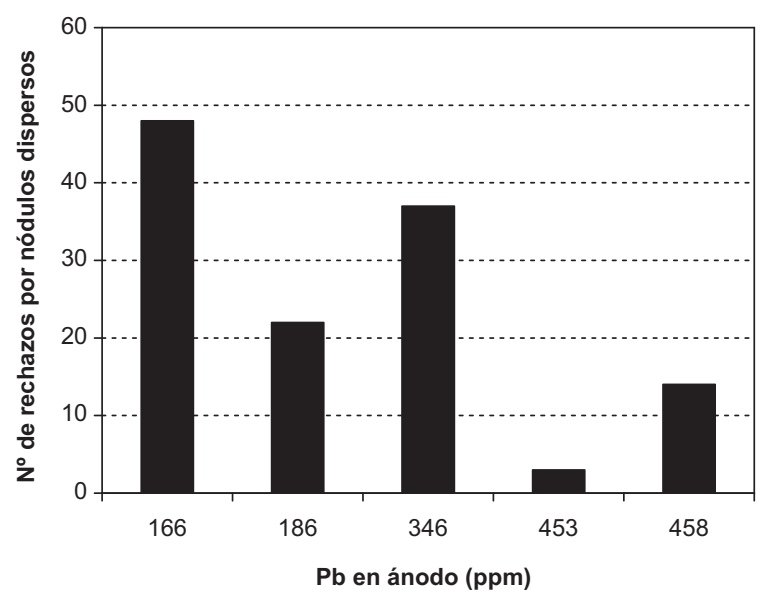

Figura 4. Relación entre el rechazo de cátodos y la cantidad de plomo en el ánodo.

Figure 4. Relation between cathode rejection and lead content in the anode. 
compuestos de $\mathrm{Pb}-\mathrm{Sb}\left(\mathrm{Pb}_{2} \mathrm{Sb}_{2} \mathrm{O}_{7}\right)$ en los barros anódicos, lo cual indujo a verificar la existencia de dicho compuesto agregando plomo a los ánodos.

Efectivamente, la presencia de bindheimita $\left(\mathrm{Pb}_{2} \mathrm{Sb}_{2} \mathrm{O}_{7}\right)$ fue más marcada en los barros producidos por aquellas campañas donde se cargaron ánodos altos en plomo (más de $1.500 \mathrm{mg} / \mathrm{l}$ de plomo), corroborando la tendencia del antimonio a formar compuestos con el plomo.

Por tanto, la presencia de plomo en los ánodos favorecería la disminución de antimonio en el electrolito, permitiendo que éste sedimente junto al plomo, debido a la alta densidad de este último, para minimizar la probabilidad de formación de nódulos en los cátodos por oclusión de partículas sobre los mismos.

\subsection{Densidad de los barros anódicos generados}

En la tabla II se muestran las densidades de los barros producidos en las campañas estudiadas en relación con la cantidad de plomo presente en los ánodos.

Una mayor cantidad de plomo en el ánodo permite tener una mayor densidad de los barros anódicos generados, lo cual permitiría hacer pensar que se obtendría una mejor calidad catódica por aumento en la sedimentación de aquellos, así como una mayor compactación en el fondo de la celda disminuyendo el levantamiento de sólidos; sin embargo, el comportamiento de las partículas del barro en relación a los rechazos no tuvo una tendencia clara para ningún tipo de ánodo estudiado ya que eran muchas las variables en juego como para llegar a un resultado determinante.

Debido a lo anterior, sólo se puede concluir que la densidad de los barros aumenta cuando la cantidad de plomo presente en el ánodo aumenta, sin presentarse una influencia clara entre los rechazos y la densidad de los barros.

\subsection{Tamaño de partícula de los barros anódicos}

Los análisis de tamaño de partícula se realizaron con las muestras dispersas en agua destilada. En la tabla III se exponen los resultados de la distribución F90 del tamaño de partícula (el $90 \%$ de las partículas se distribuyen bajo el valor especificado en micrómetros).

El tamaño de partícula de los barros anódicos estuvo relacionado directamente con la densidad de corriente aplicada. Al trabajar con densidades elevadas, para los tres tipos de ánodos, se observó que las partículas resultaron tener un tamaño mayor que al utilizar densidades bajas. Al presentarse una relación directa con la corriente, se tiene, por tanto, que mientras mayor es el tamaño de partícula menor es el rechazo de los cátodos ya que mejora la velocidad de sedimentación de las partículas.

Una posible explicación es que, al utilizar densidades de corriente elevadas existe una mayor velocidad

Tabla II. Densidad de los barros anódicos en función de la cantidad de Pb presente en los ánodos

Table II. Density of anodic slimes as a function of the lead content in the anodes

\begin{tabular}{cccc}
\hline Tipo ánodo & Barro anódico & Densidad $\left(\mathbf{g} / \mathbf{c m}^{3}\right)$ & Pb en ánodo $(\mathbf{m g} / \mathbf{l})$ \\
\hline HVL-1 & 175,74 & 4,34 & $1.539,0$ \\
HVL-2 & 137,78 & 3,80 & 557,0 \\
HVL-3 & 124,39 & 3,63 & 495,0 \\
HVL-4 & 130,39 & 3,03 & 421,0 \\
HVL-5 & 133,79 & 2,83 & 339,0 \\
HVL-6 & 139,38 & 2,97 & 542,0 \\
Vent-1 & 106,77 & 3,63 & 453,0 \\
Vent-2 & 130,52 & 3,52 & 458,0 \\
Vent-3 & 170,83 & 3,23 & 186,0 \\
Vent-4 & 133,54 & 3,12 & 346,0 \\
Vent-5 & 101,93 & 5,27 & 166,0 \\
Tte-1 & 101,62 & 3,00 & 30,0 \\
Tte-2 & 104,31 & 2,87 & 43,7 \\
Tte-3 & 110,34 & 3,00 & 34,2 \\
Tte-4 & 179,33 & 3,02 & 43,7 \\
\hline
\end{tabular}


ANALISIS DE LAS PRINCIPALES VARIABLES DE PROCESO QUE INFLUYEN EN EL RECHAZO DE LOS CÁTODOS DURANTE EL ELECTRORREFINO DEL COBRE ANALYSIS OF THE PROCESS MAIN VARIABLES INFLUENCE IN THE REJECTION OF THE CATHODES DURING COPPER ELECTROREFINING

Tabla III. Distribución de tamaño de partícula F90 en función de la densidad de corriente

Table III. F90 particle size distribution as a function of current density

\begin{tabular}{cccc}
\hline Tipo anodo & $\begin{array}{c}\text { Barro anódico } \\
\mathbf{( k g )}\end{array}$ & $\begin{array}{c}\text { Tamaño de partícula, } \\
\text { F90 }(\boldsymbol{\mu m})\end{array}$ & $\begin{array}{c}\text { Densidad de } \\
\text { corriente A/m } \mathbf{m}^{2}\end{array}$ \\
\hline HVL-1 & 175,74 & 19,97 & 260 \\
HVL-2 & 137,78 & 22,70 & 260 \\
HVL-3 & 124,39 & 44,00 & 290 \\
HVL-4 & 130,39 & 45,08 & 290 \\
HVL-5 & 133,79 & 43,57 & 310 \\
HVL-6 & 139,38 & 36,69 & 310 \\
Vent-1 & 106,77 & 29,36 & 290 \\
Vent-2 & 130,52 & 43,78 & 290 \\
Vent-3 & 170,83 & 34,38 & 290 \\
Vent-4 & 133,54 & 55,90 & 310 \\
Vent-5 & 101,93 & 49,36 & 290 \\
Tte-1 & 101,62 & 28,48 & 290 \\
Tte-2 & 104,31 & 28,48 & 290 \\
Tte-3 & 110,34 & 31,77 & 310 \\
Tte-4 & 179,33 & 42,40 & 310 \\
\hline
\end{tabular}

de disolución del ánodo, con lo cual se produce una alta cantidad de partículas pequeñas en su superficie y en la zona inmediatamente adyacente a ésta. La cantidad de partículas llega a ser tan grande, que se comienza a formar un aglomerado de éstas, de mayor tamaño, con el suficiente peso como para caer al fondo de la cuba, evitando su permanencia por un tiempo muy prolongado en el electrolito; de esta forma disminuirían los rechazos por una posible oclusión de partículas pequeñas sobre la superficie del cátodo.

Por tanto, se puede concluir que el tamaño de partícula resultó ser mayor mientras más altas fueron las densidades de corriente influyendo directamente sobre el rechazo de cátodos al minimizarlo.

\section{CONCLUSIONES}

- No se apreciaron variaciones significativas en los rechazos de cátodos al aumentar o disminuir el flujo de electrolito que ingresa a las celdas.

- Para un ciclo anódico completo, fue evidente una disminución de las partículas sólidas presentes en el electrolito, a medida que transcurrió el tiempo, para los tres tipos de ánodos.

- Las celdas del circuito experimental actúan como un sistema de decantación de sólidos.

- Perturbaciones externas como traspasos de electrolito, cortes de energía o movimiento de los elec- trodos (ánodos y cátodos), provocan un aumento de los sólidos en suspensión de entre 2 y 4 mg/l y, por tanto, en el número de cátodos rechazados.

- En general, a medida que aumentó la densidad de corriente desde 260 a $310 \mathrm{~A} / \mathrm{m}^{2}$, los rechazos disminuyeron.

- A medida que la cantidad de plomo presente en los ánodos fue mayor, los rechazos tendieron a disminuir.

- La densidad de los barros anódicos es generalmente proporcional a la cantidad de plomo presente en los ánodos.

- El tamaño de partícula de los barros anódicos se relacionó, en forma directa, con la densidad de corriente aplicada, observándose un aumento de este tamaño a medida que se trabajaba con mayor densidad de corriente $\left(310 \mathrm{~A} / \mathrm{m}^{2}\right)$. Mientras mayor resultó el tamaño de partícula de los barros, menor fue el rechazo de los cátodos.

\section{Agradecimientos}

Los autores, expresan sus agradecimientos a la Refinería Electrolítica de Ventanas de ENAMI (Empresa Nacional de Minería) y también agradecen el patrocinio prestado por la Comisión Nacional de Investigación Científica y Tecnológica a través del proyecto FONDEF D97I2035. 


\section{REFERENCIAS}

[1] T. B. Braun Et. Al., Extractive Metallurgy of Copper, Vol. 1, J. C. Yannoupoulos and J. C. Agarwel (Eds.), AIME, Port City Press, 1976.

[2] P. Mackey y A. Wraith, Miner. Process. Extractive Metall. 13 (2004) 25-37.

[3] W. C. Copper, Minerales, 43, №183, 1988.

[4] J. D. Scott, Metall. Mater. Trans. B 21 ( ) 629635.

[5] F. Noguchi, N. Lida, T. Nakamura e Y. Ueda, Metall. Revi. MMIJ 8 (1992).

[6] B. R. Conrad, Copper Hydrometallurgy, Copper 95-Cobre 95, 1995.

[7] E. N. Petkova, Hydrometallurgy 34(1994) $343-$ 358.

[8] Z. H. Gu, J. Chen y T. Z. Fahidy, Hydrometallurgy 37(1995) 149-167.

[9] G. Cifuentes Et Al., EPD Congress, TMS Annual Meeting, San Diego, EE.UU., Marzo, 1999, pp. 645-647.
[10] Z. Mubarok, I. Filzwieser Y P. Paschen, Erzmetall 58 (2005) 203-209.

[11] A. Lafront, B. Veilleux Y E. Ghali, J. Appl. Electrochem. 32 (2002) 329-337.

[12] X.Y. Liu, J. Chem. Phys. 113 (2000) 8.807-8.816.

[13] J. Dutrizac y T. T. Chen, Proc. Electrorefining and Electrowinning of Copper, 383-403.

[14] E. N. Petkova, Hydrometallurgy 46 (1997) $277-$ 286.

[15] J. B. Hiskey X. Cheng y, Metall. Trans. B 27B (1998) 53-58.

[16] D. Suárez Y F. Olson, J. Appl. Electrochem. 22 (1992) 1.002-1.010.

[17] B. Veilleuz, A. Lafront Y E. Ghali, Can. Metall. Quart. 40 (2001) 343-354.

[18] B. Veilleuz, A. Lafront Y E. Ghali, Can. Metall. Quart. 41(2002) 47-62.

[19] G. Cifuentes Et Al., Proc. Copper'99, Volumen III, Phoenix, Arizona, EE.UU., 1999, 427-435.

[20] A. Naranjo, Trabajo de Titulación, Facultad de Ingeniería, Universidad de Santiago de Chile, 2000 . 